\title{
Portal biliopathy treated with endoscopic biliary stenting
}

\author{
Sung Jin Jeon" ${ }^{1 *}$ Jae Ki Min", So Young Kwon', Jun Hyun Kim', Sun Young Moon', Kang Hoon Lee', Jeong Han Kim', \\ Won Hyeok Choe', Young Koog Cheon', Tae Hyung Kim², Hee Sun Park ${ }^{2}$ \\ 'Department of Internal Medicine, Konkuk University Medical Center, Seoul; ${ }^{2}$ Department of Radiology, Konkuk University School of \\ Medicine, Seoul, Korea
}

Portal biliopathy is defined as abnormalities in the extra- and intrahepatic ducts and gallbladder of patients with portal hypertension. This condition is associated with extrahepatic venous obstruction and dilatation of the venous plexus of the common bile duct, resulting in mural irregularities and compression of the biliary tree. Most patients with portal biliopathy remain asymptomatic, but approximately $10 \%$ of them advance to symptomatic abdominal pain, jaundice, and fever. Magnetic resonance cholangiopancreatography and endoscopic retrograde cholangiopancreatography are currently used as diagnostic tools because they are noninvasive and can be used to assess the regularity, length, and degree of bile duct narrowing. Management of portal biliopathy is aimed at biliary decompression and reducing the portal pressure. Portal biliopathy has rarely been reported in Korea. We present a symptomatic case of portal biliopathy that was complicated by cholangitis and successfully treated with biliary endoscopic procedures.

(Clin Mol Hepatol 2016;22:172-176)

Keywords: Bile duct obstruction; Portal hypertension; Endoscopic retrograde cholangiopancreatography; Biliary stent

\section{INTRODUCTION}

Portal biliopathy is defined as the development of extensive venous collateral vessels, due to obstruction of the extrahepatic portal vein, causing impediment to portal venous drainage. ${ }^{1,2}$ It develops from morphological changes of dilatation and stenosis of intrahepatic, extrahepatic and pancreatic ducts. It is also designated as cholangiopathy associated with portal hypertension, or portal cavernoma associated cholangiopathy. ${ }^{3}$ Majority of patients are asymptomatic, but rarely some experience symptoms such as abdominal pain, jaundice, and fever. ${ }^{1,2}$ Magnetic resonance cholangiopancreatography (MRCP) and endoscopic retrograde cholan- giopancreatography (ERCP) are considered to be the diagnostic tools of choice, once symptoms are manifested..$^{1-3}$ Proper treatment is required to prevent complication such as biliary sepsis in cases of symptomatic portal biliopathy. An international consensus on the management of portal biliopathy has not yet been reached. However, endoscopic method is known to be most widely used for treatment. ${ }^{3,4}$ There are few reports on radiologic findings of portal biliopathy in Korea, however, a symptomatic case was not previously reported. ${ }^{5,6}$

We reported a symptomatic case of portal biliopathy with typical clinical and radiological findings. We furthermore reviewed the diagnosis and treatment.

\section{Abbreviations: \\ CBD, common bile duct; $C T$, computer tomography; ERBD, endoscopic retrograde biliary drainage; $E R C P$, endoscopic retrograde cholangio- pancreatography; MRCP, magnetic resonance cholangiopancreatogra- phy; TIPS, transjugular intrahepatic portosystemic shunt \\ * Sung Jin Jeon and Jae Ki Min contributed equally to this work as the first authors.}

\section{Corresponding author : So Young Kwon}

Digestive Disease Center, Department of Internal Medicine, Konkuk University Hospital, Konkuk University School of Medicine, 120-1, Neungdong-ro, Gwangjin-gu, Seoul 05030, Korea

Tel: +82-2-2030-5027, Fax: +82-2-2030-5029

E-mail: sykwonmd@hotmail.com

Received : Oct. 24, 2014/ Revised : Feb. 6, 2015/ Accepted : Feb. 10, 2015

Copyright (C) 2016 by The Korean Association for the Study of the Liver

This is an Open Access article distributed under the terms of the Creative Commons Attribution Non-Commercial License (http://creativecommons.org/licenses/by-nc/3.0/) which permits unrestricted non-commercial use, distribution, and reproduction in any medium, provided the original work is properly cited. 


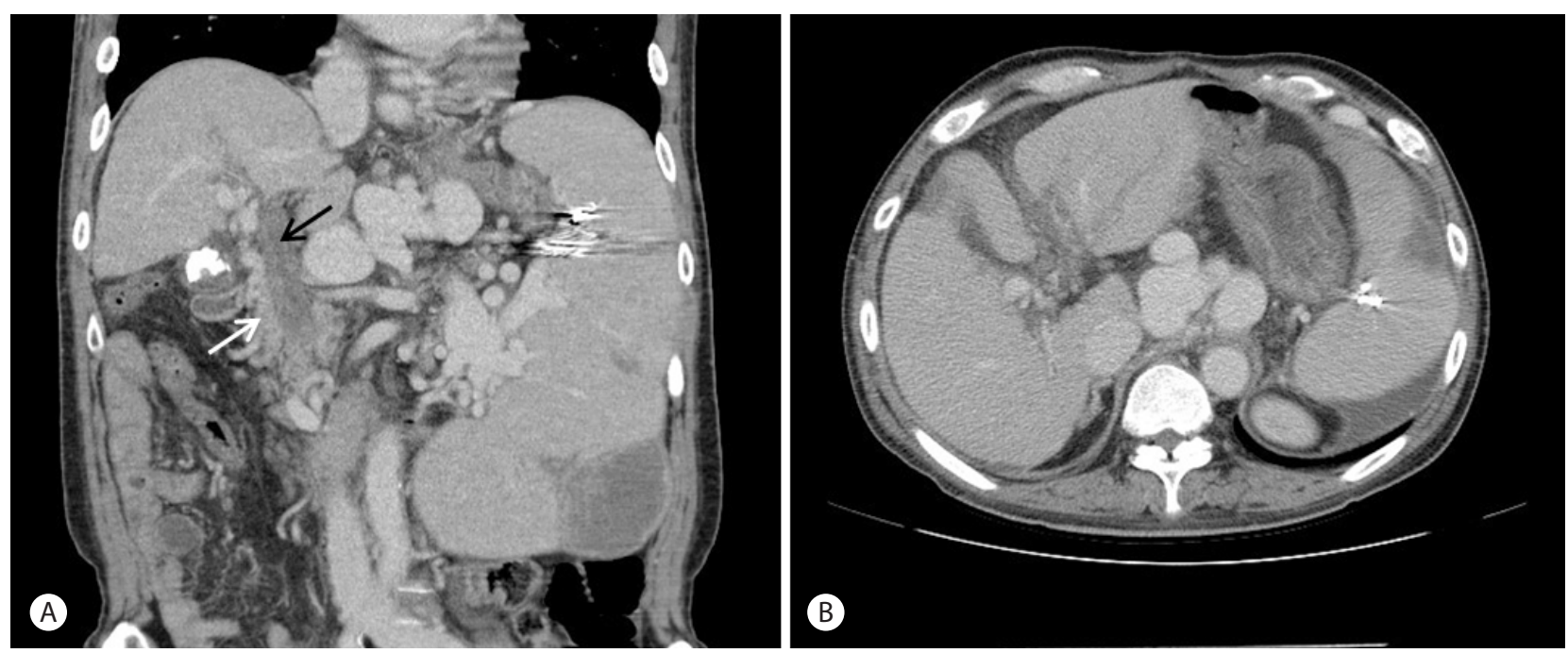

Figure 1. (A) Abdomen computed tomography (CT) scan shows the development of multiple collaterals (white arrow) due to portal vein thrombosis replacing the portal vein (black arrow). It also shows a large gallstone in the gallbladder and a splenic subcapsular organized hematoma and extensive splenic varices. (B) Abdomen CT scan shows mild left intrahepatic duct dilatation due to obstruction of the extrahepatic portal vein and liver cirrhosis, mild ascites, and splenic varices.

\section{CASE REPORT}

A 56-year-old man visited the emergency room with fever, chill, and abdominal pain. He presented with worsening jaundice since 2 days prior. The past medical history indicated that he was diagnosed with hepatitis-related liver cirrhosis and was started on an anti-viral agent since 15 years ago. He was diagnosed with a small hepatocellular carcinoma in $\mathbf{S} 2$ of the liver 8 years ago and performed trans-catheter arterial chemoembolization twice. He had been suffered from chronic portal veous thrombosis in main portal vein and portal hypertension. He had a history of embolization of a splenic arterial pseudoaneurysm with severe splenomegaly and pancytopenia 5 years ago. He had multiple hospital admissions for esophageal and gastric variceal bleeding, hepatic encephalopathy and spontaneous bacterial peritonitis. On presentation at our emergency room, he was in a febrile state and looked acutely ill with icteric sclera and abdominal tenderness and palpable abdominal mass on the left upper quadrant were found on physical examination. The vital signs on initial presentation were as follows: blood pressure of 104/66 mmHg, heart rate of 79 beats per minute, and body temperature of $38.2^{\circ} \mathrm{C}$. The initial blood test showed hemoglobin of $10.2 \mathrm{~g} / \mathrm{dL}$, white blood cell count of 3,240/ $\mathrm{L}$, platelet count of $20,000 / \mu \mathrm{L}$, prothrombin time international normalized ratio of 1.95 , albumin of $2.7 \mathrm{~g} / \mathrm{dL}$, total bilirubin of $29.4 \mathrm{mg} / \mathrm{dL}$, direct bilirubin of $20.1 \mathrm{mg} / \mathrm{dL}$, aspartate aminotransferase of $58 \mathrm{IU} / \mathrm{L}$, alanine aminotransferase of $32 \mathrm{IU} / \mathrm{L}$,

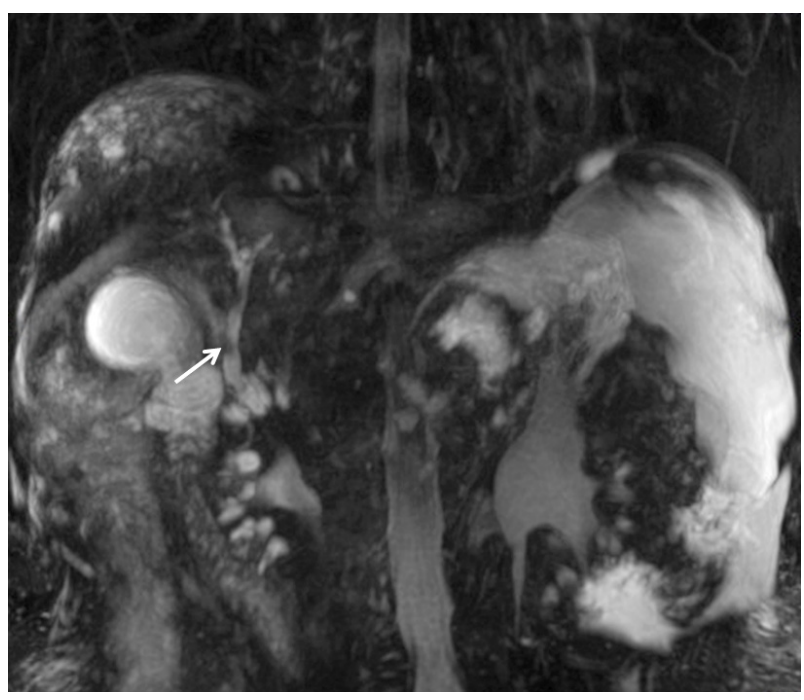

Figure 2. Magnetic resonance cholangiopancreatography shows typical filling defects in the distal common bile duct due to compression of the extrinsic cavernomatous transformation (white arrow).

alkaline phosphatase of $229 \mathrm{IU} / \mathrm{L}, \mathrm{C}$ reactive protein of $15.2 \mathrm{mg} /$ $\mathrm{dl}$, and creatinine of $1.5 \mathrm{mg} / \mathrm{dL}$. Hepatitis B e (HBe) antigen, antiHBe antibody and HBV DNA were not detected. alpha-fetoprotein was $2.3 \mathrm{ng} / \mathrm{mL}$. A plain radiograph of the chest showed no active lung lesion. However, radio-opaque gall stones were found on a plain abdominal radiograph. The impression of cholecystitis led us to perform abdominal computer tomography (CT). Abdominal CT scan showed chronic thrombosis in the main portal vein, cavernous transformation, extensive splenic varix, large gall stones and 

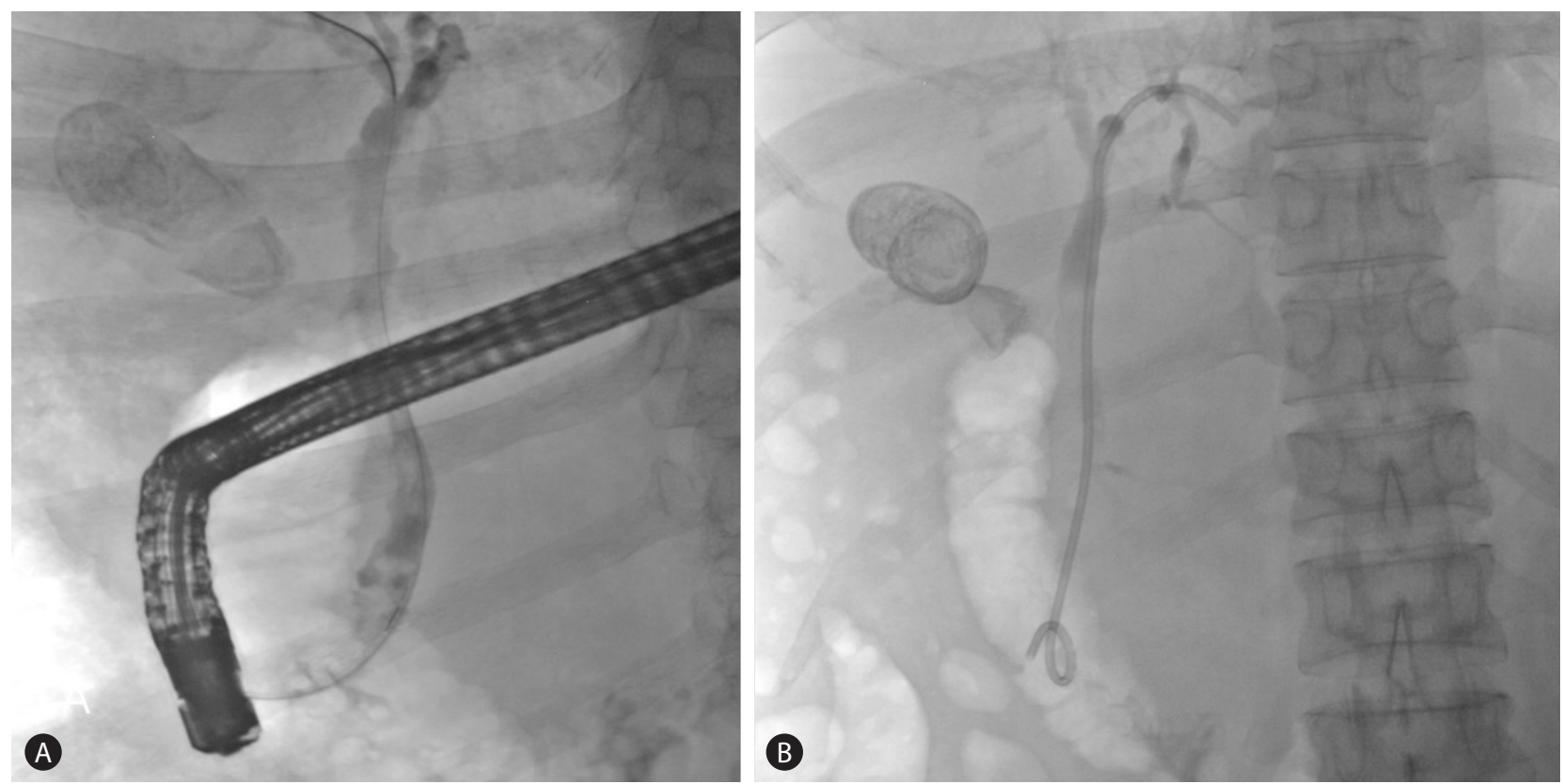

Figure 3. (A) Endoscopic retrograde cholangiogram shows filling defects in the distal common bile duct due to compression of the extrinsic cavernomatous transformation. (B) A 10-cm-long 7-Fr. double pigtail stent was inserted through the endoscopic retrograde biliary drainage (ERBD).

pericholecystic infiltration of the gallbladder (Fig. 1A). AdditionalIy, cirrhotic change of the liver, mild ascites and mild bile duct dilatation were seen (Fig. 1B). However, there were no stones in the common bile duct (CBD). These findings corresponded to early calculous cholecystitis and portal biliopathy. The patient was admitted and treated with ceftriaxone and metronidazole antibiotic therapy. MRCP was performed under suspicion of portal biliopathy or distal CBD stone. Irregular filling defect in the CBD on MRCP by cavernous transformation of the portal vein indicated portal bilopathy (Fig. 2). Stone was not identified in CBD. ERCP was performed for both the diagnosis and treatment of portal biliopathy and cholangitis. The endoscopic study revealed irregular narrowing of the CBD (Fig. 3A). Ampulla was normal and CBD stone was not observed on ERCP. Therapeutic endoscopic sphincterostomy and drainage were performed to widen the obstruction of biliary tract caused by compression. More specifically, a double pigtail stent of $7 \mathrm{Fr}$. in diameter and $10 \mathrm{~cm}$ in length was inserted through endoscopic retrograde biliary drainage (ERBD) (Fig. 3B). Fever, jaundice and abdominal pain subsided on the 2nd day after ERBD. There was a significant decrease in total bilirubin level from 29.4 to $8.9 \mathrm{mg} / \mathrm{dL}, 7$ days after ERBD. The patient had a followup blood test a month after ERBD insertion. Serum total bilirubin decreased to $2.8 \mathrm{mg} / \mathrm{dL}$ (Fig. 4). There were no symptoms including fever, chill, jaundice, abdominal pain during the follow up. Total bilirubin ranged from 1.5 to $2.0 \mathrm{mg} / \mathrm{dL}$ for 6 months until stent

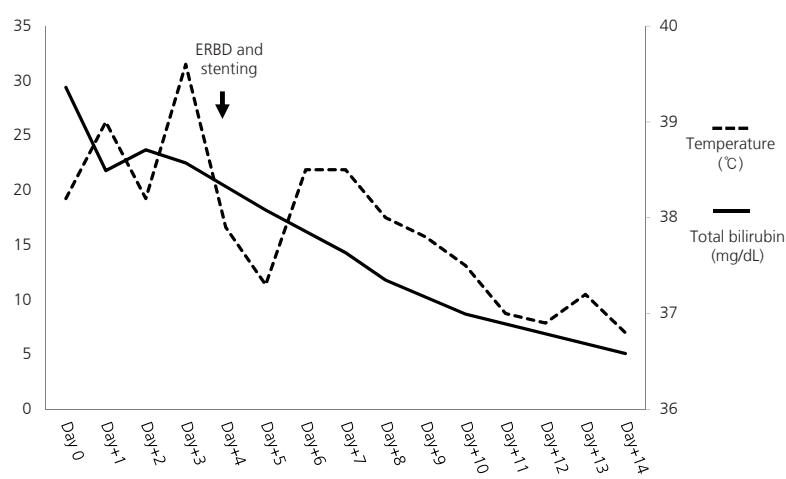

Figure 4. Clinical course of symptomatic portal biliopathy treated with biliary stenting. Day 0 , on admission. ERBD, endoscopic retrograde biliary drainage.

removal. There was no complication from ERBD. However, esophageal variceal bleeding and hepatic encephalopathy were developed during the 6 months follow-up period. The patient was hospitalized twice for bleeding control and supportive care. Furthermore, gastrointestinal bleeding from gastric antral vascular ectasia was treated with argon plasma coagulation under hospitalization.

\section{DISCUSSION}

Portal biliopathy is defined as the development of extensive ve- 
nous collateral vessels, due to the obstruction of extra- and intrahepatic ducts, causing impediment to portal venous drainage. ${ }^{1,2}$ The underlying pathogenesis of the disease starts with the compression of venous collaterals on the bile duct and prolonged portal vein thrombosis. This induces sclerosis on veins around the bile duct leading to a series of events explained by the ischemic and infective theory. ${ }^{3}$ However, this pathophysiologic mechanism is still a hypothesis without supporting experimental evidence.

Most patients remain asymptomatic and apparent symptoms with this disease are very uncommon. Approximately $5-17 \%$ of cases advance to the symptomatic stage, experiencing abdominal pain, jaundice, recurrent fever, and chilling. ${ }^{1-3}$ Patients with such symptoms are known to be comorbid with cholethiasis approximately upto $84 \%$.' The production of the stone is explained by cholestasis due to increased fibrosis and decreased motility of the biliary tract in portal biliopathy. ${ }^{3,7}$ Increase in serum bilirubin, alkaline phosphate and transaminase is caused by insufficient biliary drainage of portal biliopathy. Abnormal serum albumin level and extended prothrombin time result from prolonged biliary obstruction. ${ }^{1,2}$

Previously, non-invasive ultrasound was used for the diagnosis of portal biliopathy. It exhibits increased portal vein diameter, hyper-echogenicity on tissue of the hilum and distended paracholedochal veins. ${ }^{6}$ However, it was difficult to explore on the CBD using this modality, especially when there was no extrinsic compression by enlarged paracholedochal veins. Abdominal CT has the advantage of visualizing collateral circulation, enabling investigation on cavernous formation of the portal vein and the compressive effect of collateral circulation on the bile duct and gallbladder varices. ${ }^{5,8}$ Recently, MRCP is more often used and has become the choice non-invasive diagnostic modality for evaluating the biliary tract; it not only shows the nature and changes of intra- and extra-hepatic bile duct over time, but is also useful to rule out malignancy. ${ }^{9}$ ERCP is recommended as an invasive diagnostic tool, since it can show the irregularity, length and degree of narrowed bile duct, segmental upstream dilatation, ductal displacement, calculi, pruning of intrahepatic ducts, and the filling defects in $\mathrm{CBD} .^{10}$ The filling defect may be due to stones, varices or sclerosing cholangitis. It is also useful for differential diagnosis of recurrent pyogenic cholangitis, CBD stones with stricture, and biliary ascariasis. ${ }^{8-10}$ Endoscopic ultrasound is another diagnostic modality for portal biliopathy. Some studies have suggested that it is advantageous for the differential diagnoses of diseases including bile duct stone, pericholedochal varices and tumor. Endoscopic ultrasound is used for the diagnosis of portal biliopathy, but it is not recommended as a routine exam.'

Our patient had portal vein thrombosis causing chronic obstruction of the extrahepatic portal vein. He suffered from variceal bleeding due to portal hypertension but had no biliary symptoms before admission. Symptoms including recurrent fever, chill, jaundice, and abdominal pain developed over time. Serum laboratory and image studies were conducted under suspicion of cholangitis and cholecystitis. CT revealed typical development of multiple collateral vessels, gallstone and portal cavernoma presumably caused by portal vein thrombosis, however CBD stone was not observed. MRCP was followed immediately and showed typical filling defects on the distal bile duct due to compression from extrinsic cavernomatous transformation without CBD stone. MRCP is a non-invasive diagnostic modality for detection of small biliary stone with high sensitivity and specificity (>90\%). ${ }^{11}$ Therefore, we could exclude choledocholithiasis as a cause of biliary obstruction. However, there might be a possibility of passage of small stone of CBD.

Asymptomatic portal biliopathy does not require treatment. ${ }^{1,4}$ On the other hand, treatment to reduce symptoms and to prevent complication such as cholecystitis, cholangitis, chronic obstructive jaundice, biliary sepsis and biliary cirrhosis, is recommended in symptomatic cases. ${ }^{1,2,4}$ Endoscopic treatment should be considered as an initial therapeutic modality when there are symptoms caused by biliary stricture or obstruction, since it effectively alleviates symptoms of biliary obstruction. ${ }^{3,4,2}$ Endoscopic treatment includes balloon catheter dilation, endoscopic papillotomy and stent insertion. Endoscopic treatment also has some disadvantages; it requires regular follow-up exams for monitoring stent migration and for a replacement of stent when occluded. A case study on endoscopic stent indicated that patients with prolonged indwelling stent suffered from recurrent cholangitis. ${ }^{13}$ Therefore, screening exams should be performed periodically. As mentioned earlier, though endoscopic treatment is the first choice for portal biliopathy, a favorable outcome may not always be achieved. ${ }^{2,12}$ Transjugular intrahepatic portosystemic shunt (TIPS) could be considered when symptoms persist even after endoscopic biliary decompression. Biliary intestinal bypass is the next treatment choice, when TIPS fails to significantly reduce symptoms. ${ }^{12}$ Surgical intervention should be considered in patients if the symptoms from the stricture or obstruction of bile duct continue, or when regular follow-up exams is expected to be difficult for a patient. ${ }^{3,12}$

In this case, endoscopic treatment was considered as the initial therapeutic modality since our patient experienced symptoms of 
biliary obstruction. The subject successfully received balloon catheter dilation, endoscopic papillotomy and stent insertion and was discharged from the hospital. There was no complication from the procedure itself, such as recurrent cholangitis or stent obstruction. The stent was removed 6 months after the ERBD insertion. Despite successful endoscopic treatment on portal biliopathy, complications from portal hypertension including hepatic encephalopathy, and variceal bleeding and GAVE bleeding could not be avoided in the patient. Complications of portal hypertension or cirrhosis should be carefully monitored and promptly managed during the follow-up in portal biliopathy patients.

In conclusion, when a patient with portal hypertension and prominent extrahepatic collaterals experiences jaundice, fever, and abdominal pain, portal biliopathy should be considered in the differential diagnosis. Abdominal CT, MRCP, or ERCP are the recommended differential diagnostic procedures. Once portal biliopathy is confirmed, the treatment plan should be established according to each patient's clinical symptoms and disease status. Endoscopic biliary decompression should be considered as the initial management. However, this treatment is limited since it does not radically resolve primary portal hypertension and complications of hepatic decompensation.

\section{Conflicts of Interest}

The authors have no conflicts to disclose.

\section{REFERENCES}

1. Chattopadhyay S, Nundy S. Portal bilipathy. World J Gastroenterol 2012;18:6177-6182.
2. Chandra R, Kapoor D, Tharakan A, Chaudhary A, Sarin SK. Portal biliopathy. J Gastroenterol Hepatol 2001;16:1086-1092.

3. Khan MR, Tariq J, Raza R, Effendi MS. Portal hypertensive biliopathy: review of pathophysiology and management. Trop Gastroenterol 2012;33:173-178.

4. Sezgin O, Oğuz D, Altintaş E, Saritaş U, Sahin B. Endoscopic management of biliary obstruction caused by cavernous transformation of the portal vein. Gastrointest Endosc 2003;58:602-608.

5. Choi JH, Chung WJ. Portal bilopathy. Korean J Gastroenterol 2012;59:445-447.

6. Kim TO, Kim GH, Hwang SY, Lee SY, Heo J, Kang DH, et al. Clinical features and imaging findings of portal biliopathy. Korean J Med 2006;70:518-526.

7. Harmanci O, Bayraktar Y. How can portal vein cavernous transformation cause chronic incomplete biliary obstruction? World I Gastroenterol 2012;18:3375-3378.

8. Ozkavukcu E, Erden A, Erden I. Imaging features of portal biliopathy: frequency of involvement patterns with emphasis on MRCP. Eur J Radiol 2009;71:129-134.

9. Condat B, Vilgrain V, Asselah T, O'Toole D, Rufat P, Zappa M, et al. Portal cavernoma-associated cholangiopathy: a clinical and MRcholangiography coupled with MR portography imaging study. Hepatology 2003;37:1302-1308.

10. Besa C, Cruz JP, Huete A, Cruz F. Portal biliopathy: a multitechnique imaging approach. Abdomen Imaging 2012;37:83-90.

11. Guarise A, Baltieri S, Mainardi P, Faccioli N. Diagnostic accuracy of MRCP in choledocholithiasis. Radiol Med 2005;9:239-251.

12. Suárez V, Puerta A, Santos LF, Pérez JM, Varón A, Botero RC. Portal hypertensive biliopathy: A single center experience and literature review. World J Hepatol 2013;5:137-144.

13. Dilawari JB, Chawla YK. Pseudosclerosing cholangitis in extrahepatic portal venous obstruction. Gut 1992;33:272-276. 